\title{
AVALIAÇÃO DAS CARACTERÍSTICAS HIDRÁULICAS DO MICRODIFUSOR AUTOCOMPENSANTE DAN JET 7200
}

\author{
RICARDO AUGUSTO DA SILVA ${ }^{1}$ \\ ANTONIO MARCIANO DA SILVA ${ }^{2}$
}

\begin{abstract}
RESUMO - Com a finalidade de suprir com informações as necessidades dos projetistas de irrigação, foi realizado este trabalho de avaliação de um microdifusor autocompensante DAN JET 7200, submetido a testes no Laboratório de Irrigação do Departamento de Engenharia da Universidade Ferderal de Lavras - MG (UFLA). Todos os testes realizados consistiram da aquisição de valores de vazões a diferentes pressões. Para isso, foram utilizados vinte emissores, procurando-se avaliar os seguintes parâmetros: curva característica dos emissores, coeficiente de variação de fabricação
\end{abstract}

(CVF), uniformidade de vazão, uniformidade de distribuição, perfil de distribuição da precipitação em relação à distância da haste de sustentação e alcance dos jatos. Esses testes evidenciaram maior eficiência da membrana reguladora para o intervalo de pressão proposto pelo fabricante. Os emissores ainda apresentaram bom coeficiente de variação de fabricação, excelente uniformidade de vazão para todas as pressões testadas, péssima uniformidade de distribuição da precipitação e maior concentração e alcance de jatos em uma faixa entre 1,20 e $2,10 \mathrm{~m}$.

TERMOS PARA INDEXAÇÃO: Irrigação, emissor e características hidráulicas.

\section{EVALUATION OF HYDRAULIC CHARACTERISTICS OF THE DAN-JET 7200 FLOW-COMPENSATED MICROSPRINKLER}

\begin{abstract}
To provide information required by designers of irrigation systems, evaluation of the DANJET 7200 flow-compensated micro sprinkler was carried out. The sprinklers were submitted to indoor tests at the Hydraulic Lab of the Engineering Department at the Federal University of Lavras, Brazil. During the tests the sprinkler was characterized and the working pressure range proposed by the manufacturer evaluated. This was achieved by determining the following parameters: sprinkler characteristic curve,
\end{abstract}

coefficient of variation, discharge uniformity, distribution uniformity, radial profile of precipitation, and wetted radius. Results from these tests showed that the pressure regulator membrane presents a better performance when working in the range of pressures recommended by the manufacturer. The sprinkler achieved a good classification, in regard the coefficient of variation, with good discharge uniformity in all situations, poor precipitation distribution uniformity, and jet concentration in the range $1.20-2.10 \mathrm{~m}$.

INDEX TERMS: Irrigation, emitter and hydraulics characteristics.

\section{INTRODUÇÃO}

Para satisfazer às necessidades de uma agricultura moderna, novos equipamentos são fabricados com os mais variados tipos de máquinas e materiais. O surgimento de novos equipamentos implica na necessidade de determinação de suas características estruturais e de análise de seu desempenho. Dos componentes de um sistema de irrigação localizada, os emissores constituem-se na peça principal. Portanto, a escolha de um e- missor deve seguir a avaliação detalhada de suas características.

Para Botrel (1984), a relação entre vazão e pressão na entrada do microaspersor, a perda de carga localizada na inserção da linha lateral, o alcance do jato, o diâmetro, a forma dos bocais e a intensidade de precipitação constituem as principais características hidráulicas do microaspersor.

Segundo Solomon e Keller (1978), variações nas dimensões dos emissores sempre ocorrerão e não de-

\footnotetext{
1. Mestre em Engenharia Agrícola pela UNIVERSIDADE FEDERAL DE LAVRAS/UFLA - Caixa Postal 37 37200-000 - Lavras, MG.

2. Professor Titular da UFLA.
} 
pendem apenas do controle de qualidade dos materiais de construção, mas também de sua geometria.

A vazão de um emissor relaciona-se diretamente com a carga hidráulica atuante na sua entrada e pode ser representada, segundo Keller e Karmeli (1975), pela seguinte equação:

$$
\mathrm{q}=\mathrm{k} \cdot \mathrm{H}^{\mathrm{x}}
$$

em que:

q - vazão do emissor $(1 / h)$;

$\mathrm{k}$ - constante de proporcionalidade que caracteriza cada emissor;

$\mathrm{H}$ - pressão de serviço do emissor $(\mathrm{kPa})$;

$\mathrm{x}$ - expoente de descarga do emissor que caracteriza $\mathrm{o}$ regime de fluxo.

Segundo Pizarro (1990), um gotejador autocompensante perfeito seria representado por uma equação do tipo $\mathrm{Q}=\mathrm{kH}^{\mathrm{x}}$, em que o expoente $\mathrm{x}$ seria zero, ou seja, a vazão se manteria constante em toda a linha.

Keller e Karmeli (1975), citados por Boman (1989), introduziram o coeficiente de variação de fabricação (CVF) como uma medida estatística da variação de fabricação dos emissores para irrigação. Para Pizarro (1990), o coeficiente de variação (CV ou CVF) é o parâmetro que melhor se adequaria à medida de dispersão.

O coeficiente de variação de fabricação (CVF) é obtido pela expressão:

$$
\mathrm{CVF}=\frac{\sigma f(q)}{\mathrm{qmf}}
$$

em que:

CVF - coeficiente de variação de fabricação;

of(q) - desvio padrão da vazão a uma pressão de referência;

qmf - vazão média a uma pressão de referência;

De acordo com Nakayama e Bucks (1986), informações referentes à vazão dos emissores e sua uniformidade são essenciais para o dimensionamento dos sistemas de irrigação localizada.

A mais popular medida da uniformidade de irrigação (Equação 3) é o coeficiente de uniformidade de Christiansen (1942), amplamente utilizado na determinação da uniformidade em função da precipitação nas irrigações convencionais e adaptado para a irrigação localizada, utilizando dados em função da vazão.

$$
\mathrm{CUC}=100\left[1-\frac{\sum_{\mathrm{i}=1}^{\mathrm{n}}\left|\mathrm{q}_{\mathrm{i}}-\mathrm{q}_{\mathrm{m}}\right|}{\mathrm{N} \cdot \mathrm{q}_{\mathrm{m}}}\right]
$$

em que:

CUC $=$ coeficiente de uniformidade de Christiansen, em porcentagem;

$\mathrm{q}_{\mathrm{i}}=$ vazão de cada emissor $\left(\mathrm{L}^{3} \mathrm{~T}^{-1}\right)$;

$\mathrm{q}_{\mathrm{m}}=$ média das vazões observadas $\left(\mathrm{L}^{3} \mathrm{~T}^{-1}\right)$;

$\mathrm{N}=$ número de emissores.

No entanto, de acordo com Pizarro (1990), em irrigação localizada, utilizam-se critérios mais exigentes. Esse autor defende a utilização do coeficiente de uniformidade de Criddle (1956), (PE), para definir a uniformidade de vazão (equação 4).

$$
\mathrm{PE}=100 \frac{\overline{q_{25}}}{\mathrm{q}_{\mathrm{m}}}
$$

em que:

$\mathrm{PE}=$ eficiência padrão de Criddle em porcentagem;

$\overline{\mathrm{q}_{25}}=$ média das $25 \%$ menores vazões coletadas, $\left(\mathrm{L}^{3} \mathrm{~T}^{-1}\right)$;

Realizou-se este trabalho com o objetivo de avaliar em laboratório as características hidráulicas do microdifusor autocompensante DAN JET 7200.

\section{MATERIAL E MÉTODOS}

A caracterização hidráulica do emissor foi realizada no Laboratório de Irrigação do Departamento de Engenharia, da Universidade Federal de Lavras-MG.

$\mathrm{O}$ emissor avaliado foi o microdifusor autocompensante DAN JET 7200, bocal violeta (diâmetro $4,53 \mathrm{~mm}$ ), com ângulo de cobertura de $300^{\circ}$, vazão nominal $47 \mathrm{Lh}^{-1}$ e raio de alcance de $3,7 \mathrm{~m}$ a uma pressão de $1 \mathrm{kPa}$, com difusor estriado formando doze jatos, fabricado em plástico de alta resistência, acoplado a uma haste de $0,40 \mathrm{~m}$, que o sustenta a $0,20 \mathrm{~m}$ do solo. A ligação do emissor à linha de distribuição é feita por meio de um tubo flexível de $4 \mathrm{~mm}$ de diâmetro e 0,60 $\mathrm{m}$ de comprimento. A conexão na linha é feita por meio de encaixe de pressão.

A pressão foi medida com dois manômetros tipo bourdon e controlada por dois registros, um situado na saída da bomba e outro, em um ponto próximo à entrada do emissor. As distâncias entre as conexões respeitaram as normas da ABNT (1986) referentes a avaliações de emissores.

Para obtenção da curva característica vazão versus pressão e a variação devida ao processo de fabricação, foram obtidos dados de vazão do microdifusor operando sob diferentes pressões.

Os emissores foram ensaiados nas seguintes pressões: 49; 98; 147; 196; 245; 294 e $343 \mathrm{kPa}$, que 
correspondem, respectivamente, às pressões de 5,10 , $15,20,25,30$ e $35 \mathrm{mca}$, e o fabricante recomenda o funcionamento desse no intervalo de pressão 98,0 a $294 \mathrm{kPa}$. O tempo de coleta foi de cinco minutos.

A uniformidade de distribuição de água foi avaliada com o auxílio de pluviômetros dispostos em linhas sob seis dos doze jatos da água do emissor, previamente sorteado. Os coletores foram espaçados entre si de 0,30 $\mathrm{m}$ até uma distância de $3 \mathrm{~m}$ da haste de sustentação do emissor. Foram utilizados como pluviômetros coletores cilíndricos de plástico de $0,103 \mathrm{~m}$ de altura e $0,0812 \mathrm{~m}$ de diâmetro. Nesse teste, foram utilizados cinco emissores, submetidos às pressões de 98, 196 e $294 \mathrm{kPa}$, e colocados em funcionamento pelo período de uma hora. A água contida nos pluviômetros foi pesada em balança de precisão (miligramas) e, posteriormente, convertida em lâminas de precipitação.

Para o teste de uniformidade de vazão, foram utilizados os dados obtidos no teste de pressão e vazão e, para o teste de uniformidade de distribuição de precipitação, os dados utilizados foram provenientes de uma malha de pluviômetros espaçados, eqüidistante entre si de $0,25 \mathrm{~m}$, dispostos sob os jatos.

Para avaliar a uniformidade de vazão, foram utilizados os Coeficientes de Uniformidade de Christiansen (CUC) e de Criddle (PE) e para a uniformidade de distribuição, o coeficiente de Christiansen (CUC).

\section{RESULTADOS E DISCUSSÃO}

\section{Equação característica do emissor}

Com os dados de pressão e vazão, foram geradas as equações características e as curvas para o microdifusor, (Figuras 1 e 2) para os intervalos de pressão 49 a $343 \mathrm{kPa}$ e 98 a $294 \mathrm{kPa}$, respectivamente.

Os valores baixos do expoente $\mathrm{x}$ devem-se ao dispositivo autocompensante. Com base nas equações, observa-se maior eficiência da membrana autocompensante no intervalo de pressão 98 a $294 \mathrm{kPa}$, recomendado pelo fabricante.

\section{Coeficiente de variação}

Levando-se em conta que as condições de teste foram iguais para todos os emissores ensaiados, as diferenças de vazões ocorridas são devidas ao pro- cesso de fabricação. Os coeficientes de variação de vazão, para as diferentes pressões analisadas, encontram-se na Tabela 1 . Os menores valores de CV encontrados foram os relacionados com as menores pressões de teste.

TABELA 1 - Coeficientes médios de variação de vazão do microdifusor autocompensante DAN JET 7200, para as pressões analisadas e para o intervalo de pressão recomendado pelo fabricante.

\begin{tabular}{cc}
\hline Pressão & C.V. \\
kPa & \% \\
\hline 49 & 5,75 \\
98 & 6,00 \\
147 & 8,07 \\
196 & 10,02 \\
245 & 9,26 \\
294 & 8,30 \\
343 & 9,04 \\
\hline C.V médio & $\mathbf{8 , 0 6}$ \\
\hline Média & $\mathbf{8 , 3 3}$ \\
\hline
\end{tabular}

Pode ser observado, na classificação dos emissores quanto ao processo de fabricação (Tabela 2), que os mesmos comportam-se dentro de uma faixa ainda aceitável de acordo com as fontes de classificação.

Precipitação em função da distância em relação à haste de sustentação do emissor

A distribuição da precipitação em função da distância, para o intervalo de pressão recomendado pela fabricante, pode ser observada na Figura 3. As maiores precipitações foram encontradas entre 1,20 e 2,10 m de distância . Os maiores valores de precipitação encontrados para as menores pressões devem-se à menor dissipação de energia no contato entre jato e placa difusora, fazendo com que os jatos de água fiquem mais localizados. 


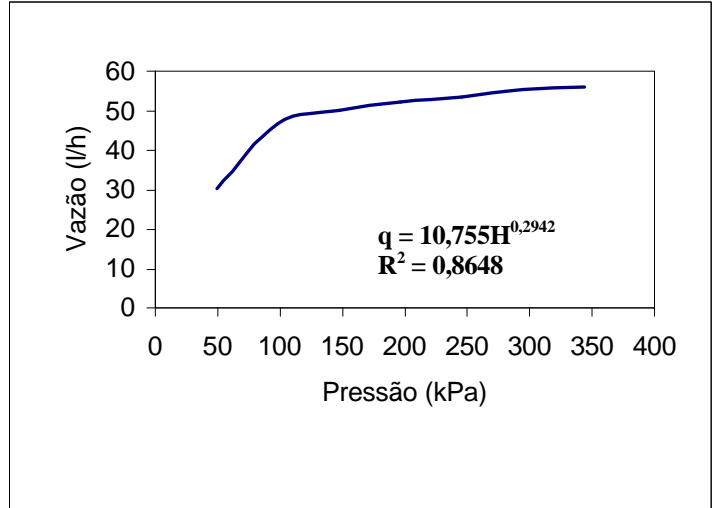

FIGURA 1 - Relação vazão x pressão para o microdifusor autocompensante DAN JET 7200, para pressão entre 49 e $343 \mathrm{kPa}$.

TABELA 2 - Classificação dos emissores quanto ao coeficiente de variação.

\begin{tabular}{lcc}
\hline \multicolumn{1}{c}{ Fontes } & $\begin{array}{c}\mathbf{4 9} \mathbf{a ~ 3 4 3} \\
\mathbf{k P a}\end{array}$ & $\begin{array}{c}\mathbf{9 8} \mathbf{a ~ 2 9 4} \\
\mathbf{k P a}\end{array}$ \\
\hline ABNT (1986) & Bom & Bom \\
$\begin{array}{l}\text { Nakayama e Bucks } \\
\text { (1986) }\end{array}$ & Médio & Médio \\
$\begin{array}{l}\text { Hardie Irrigation } \\
\text { Normas UNE }\end{array}$ & Médio & Médio \\
$\begin{array}{l}\text { Irrigation United States } \\
\text { Dept. of Agric }\end{array}$ & Marginal & Marginal \\
\hline
\end{tabular}

\section{Uniformidade de vazão e de distribuição}

A uniformidade de vazão dos emissores, obtida pelos coeficientes de Chistiansen (CUC) e de Criddle (PE), pode ser observada na Tabela 3.

Os valores dos coeficientes de uniformidade encontram-se em faixas que, na literatura, são consideradas como adequadas.

Valores baixos de uniformidade de distribuição da precipitação foram encontrados. Essa é uma característica dos emissores, que, de certo modo, é compensado, na irrigação localizada, pela redistribuição da

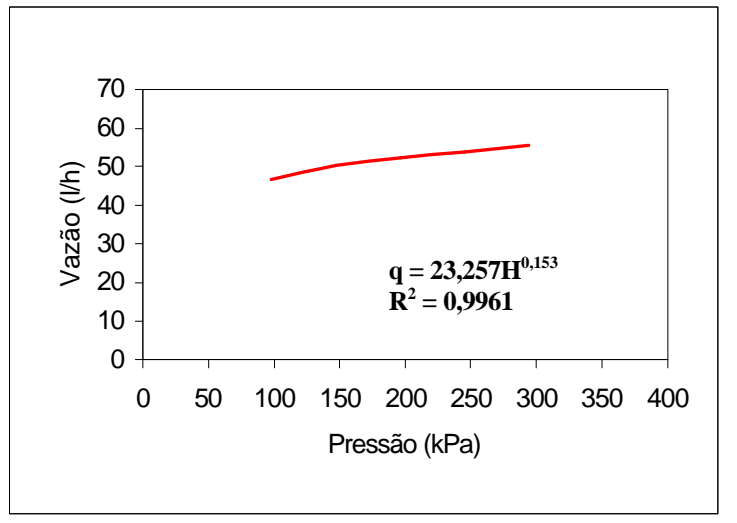

FIGURA 2 - Relação vazão x pressão para o microdifusor autocompensante DAN JET 7200, para pressão entre, 98 e $294 \mathrm{kPa}$.

água no perfil do solo. Nas Figuras 4, 5, e 6 evidenciam-se os perfis de distribuição de água do microdifusor, submetido às pressões de 98, 196 e $294 \mathrm{kPa}$. Pode-se observar claramente o posicionamento dos doze jatos de água e a área molhada em sua extremidade.

TABELA 3 - Valores de coeficientes de uniformidade de vazão obtidos para as diferentes pressões testadas.

\begin{tabular}{ccc}
\hline Pressões kPa & CUC (\%) & PE (\%) \\
\hline 49 & 95,95 & 93,44 \\
147 & 96,40 & 92,80 \\
196 & 93,82 & 90,56 \\
245 & 94,40 & 88,99 \\
294 & 97,32 & 90,35 \\
343 & 95,41 & 90,84 \\
\hline Média & 94,97 & 90,07 \\
\hline
\end{tabular}




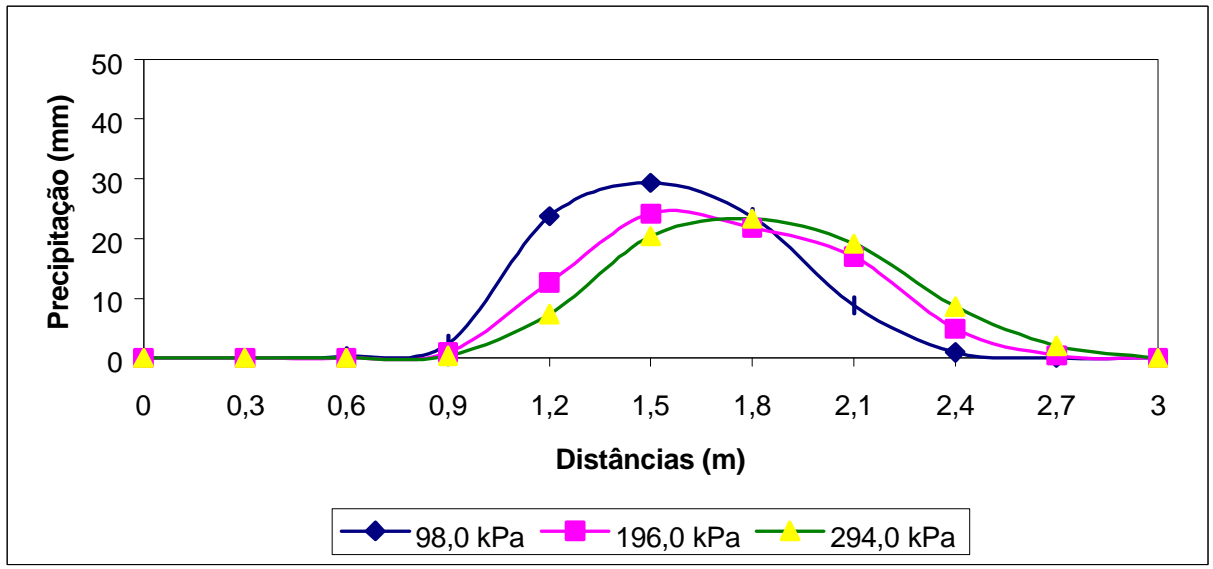

FIGURA 3 - Perfil de distribuição de água em relação à distância do microdifusor autocompensante DAN 7200, para as pressões de 98,196 e $294 \mathrm{kPa}$.

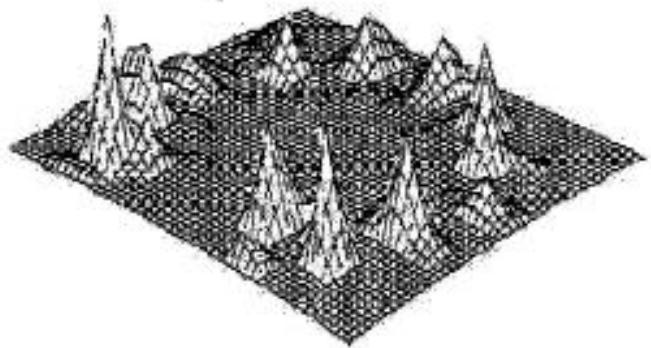

FIGURA 4 - Perfil de distribuição de água do microdifusor autocompensante DAN JET 7200, a pressão de $98 \mathrm{kPa}$.

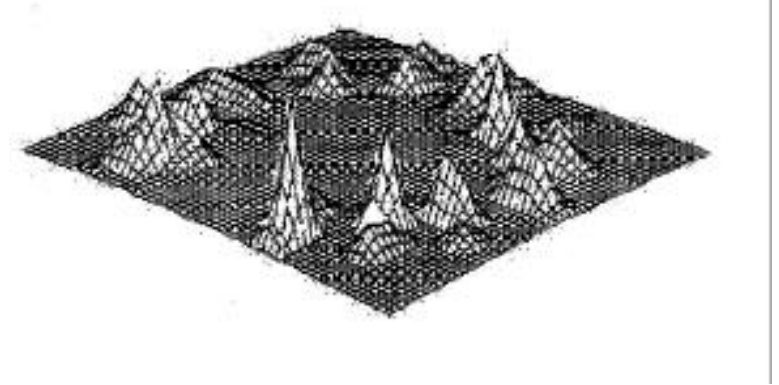

FIGURA 5 - Perfil de distribuição de água do microdifusor autocompensante DAN JET 7200, a pressão de $196 \mathrm{kPa}$.

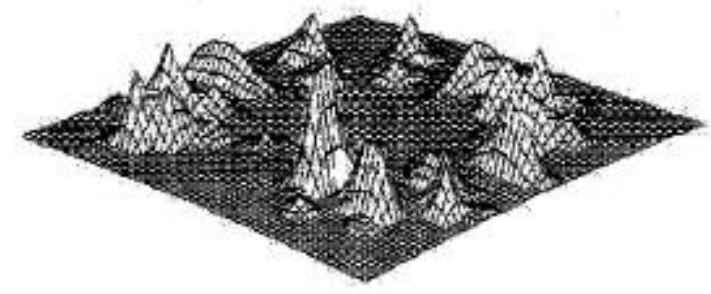

FIGURA 6 - Perfil de distribuição de água do microdifusor autocompensante DAN JET 7200, a pressão de $294 \mathrm{kPa}$. 


\section{CONCLUSÕES}

Nas condições de realização dos testes, o microdifusor autocompensante DAN JET 7200

a) apresentou uma melhor eficiência de funcionamento da membrana auto-reguladora de pressão para o intervalo de pressão proposto pelo fabricante;

b) obteve uma classificação razoável quanto ao coeficiente de variação;

c) os maiores valores de precipitação foram encontrados entre 1,20 e $2,10 \mathrm{~m}$ de distância, em relação à haste de sustentação;

d) teve a maior concentração de jatos na faixa de 1,80 a $2,40 \mathrm{~m}$; e

e) apresentou excelentes valores de coeficiente de uniformidade de vazão e péssimos de uniformidade de precipitação.

\section{REFERÊNCIAS BIBLIOGRÁFICAS}

ASSOCIAÇÃO BRASILEIRA DE NORMAS TÉCNICAS. Projeto 12:02:08-020: sistema de irrigação localizada: avaliação do desempenho características operacionais. Rio de Janeiro, 1986.

BOMAN, B. J. Emitter and spaghetti tubing effects on microsprinkler flow uniformity. Transactions of The ASAE, Saint Joseph, v. 32, n. 1, p. 168-172, 1989.
BOTREL, T. A. Hidráulica de microaspersores e linhas laterais para irrigação localizada. 1984. $78 \mathrm{f}$. Dissertação (Mestrado em Irrigação e Drenagem) - Escola Superior de Agricultura "Luiz de Queiroz", Piracicaba, 1984.

CHRISTIANSEN, J. E. Irrigation by splinkling. Berkeley: University of California, 1942. 124 p. (Bulletim, 670).

CRIDDLE, W. D. Methods for evaluating irrigations systems agriculture. [S.1.]: Hand book, 1956. 82 p.

KELLER, J.; KARMELI, D. Trikler irrigation desing. Glendora: Rain Bird Sprinkler Manufacturing, 1975. $182 \mathrm{p}$.

NAKAYAMA, F. S.; BUCKS, D. A. Trickle irrigation for crop production. Phoenix: Elservier, 1986. $383 \mathrm{p}$.

PIZARRO, F. Riegos localizados de alta frecuencia: goteo, microaspersion, exudación. 5. ed. Madri: Mundi, 1990. $461 \mathrm{p}$.

SOLOMOM, K.; KELLER, J. Trickler irrigation uniformity and efficiency. Jornal of the Irrigation e Drainagem Division, Ann Arbor, v. 115, p. 293-306, Sept. 1978. 